PSS PROCEEDINGS

\title{
LHCb: status and physics results
}

Irina Machikhiliyan ${ }^{* \dagger}$

Laboratoire d'Annecy-le-Vieux de Physique des Particules, Annecy-le-Vieux, France

E-mail: Irina.Machikhiliyan@cern.ch

LHC on the March,

November 16-18, 2011

Protvino, Moscow region, Russian Federation

* Speaker.

${ }^{\dagger}$ For the LHCb Collaboration 


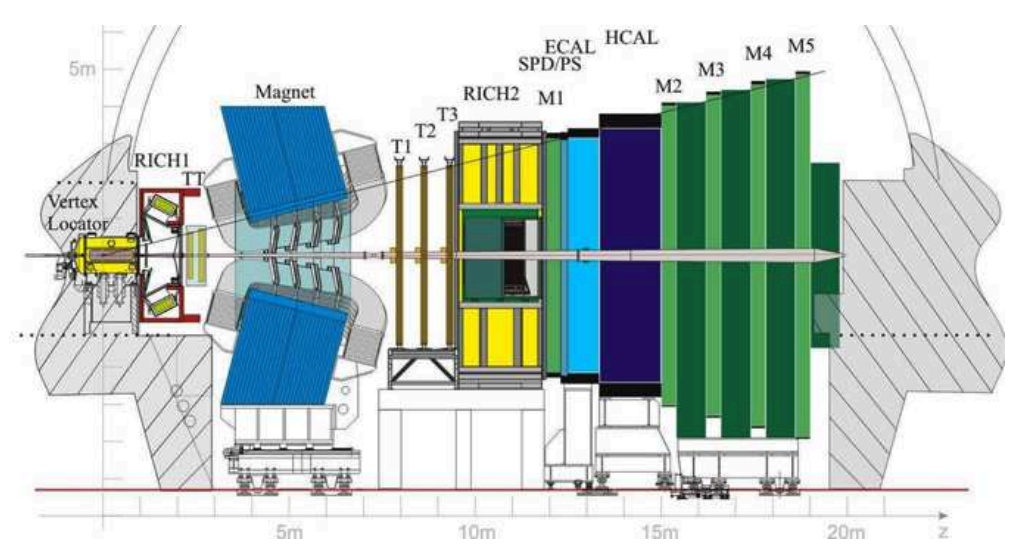

Figure 1: Layout of the LHCb apparatus.

\section{Introduction}

The LHCb experiment [1] is dedicated to heavy-flavour sector studies exploiting the unique potential of the LHC accelerator, which combines large $b \bar{b}$ - and $c \bar{c}$-pairs production cross-sections and high luminosity. LHCb physics program includes the following main topics: (i) searches of new physics beyond the Standard Model (SM) via rare $B$-decays; (ii) studies of $C P$-violation in neutral $B$-systems; (iii) charm physics and (iv) production and spectroscopy (inclusive particle production, jets, searches of exotic states, quarkonia, etc).

\section{LHCb apparatus}

At LHC, heavy quark pairs are boosted together in the same direction which leads to the forward single arm spectrometer design of the apparatus with solid angle coverage from 1.9 to 4.9 in units of pseudorapidity (see Fig. 1). Such design means high occupancies and significant radiation doses in the central area of the detector. This issue is solved by a proper choice of granularities and materials as well as the introduction of a lumi-leveling system, which decreases the luminosity $L$ by controlling the proton bunches overlap. The design value of $L$ is $2 \times 10^{32} \mathrm{~cm}^{-2} \mathrm{~s}^{-1}$, while the average number of interactions per bunch crossing $\mu$ is set to be 0.4. Another challenge is high background; only 1 out of approximately 200 collisions contains $b$-quarks, thus the experiment requires an efficient trigger system, sensitive to many final states, as well as a powerful high bandwidth data acquisition system.

\subsection{The Tracking System}

A high-precision tracking system with an excellent vertex, impact parameter and momentum resolution is a prerequisite for $\mathrm{LHCb}$. This is especially important to study the rapidly oscillating $B_{s}^{0}$-system, where good proper time resolution is required. The LHCb tracking system includes a vertex locator (VELO) for the reconstruction and the separation of primary and secondary vertices, a dipole magnet and several planes of tracker stations. 
The VELO consists of $42 r$ - and $42 \phi$-measuring silicon microstrip sensors; their pitch size varies from $37 \mu \mathrm{m}$ up to $98 \mu \mathrm{m}$ depending on the sensor type and the distance from the beam axis. Measurements are conducted as close to the interaction point IP as possible (up to $8 \mathrm{~mm}$ ), therefore to avoid damaging of the sub-detector during the periods of beams injection the sensors are retractable. Four extra sensors, installed upstream the IP, form the pile-up system, which purpose is to veto crossings with multiple interactions.

The silicon tracker (ST) employs silicon microstrip detectors with $200 \mu \mathrm{m}$ pitch which are arranged in stations of four stereo-layers with two central layers rotated by $\pm 5^{\circ}$. Two stations are situated upstream the magnet and are used to improve the reconstruction of long-lived particles and to compensate for the multi-scattering in detector material. Another three stations serve as inner sections of three tracker planes downstream the magnet, while their peripheral areas are composed of the outer tracker (OT) chambers. The latter is made of straw tubes of $\sim 5 \mathrm{~mm}$ diameter filled with $\mathrm{Ar}-\mathrm{CO}_{2}$ mixture ensuring fast drift time within $50 \mathrm{~ns}$. Similar to ST, each OT station has four stereo-layers with $\pm 5^{\circ}$ stereo-angle values for the two central layers.

The performance of the LHCb tracking system has been studied with the first data and proved to be complying with the design requirements. In particular, the most crucial parameter - proper time resolution for $B_{s}^{0}-\bar{B}_{s}^{0}$ oscillations studies with $J / \Psi$ in final state - was found to be $50 \mathrm{fs}$ with $2 \%$ systematic error [2].

\subsection{The Particle Identification System}

The particle identification system includes two ring imaging Čerenkov counters(RICHes) for $\pi / K / p$ separation; a calorimeter system (CALO) to provide fast trigger on energetic e $/ \gamma / \pi^{0} / h$, to perform $e / h$ separation and to measure the parameters of neutral particles; a muon system which provides muon trigger and offline identification of muons.

The RICH1, which is situated upstream the magnet, employs two types of radiator: silica aerogel to cover low momenta range from $1 \mathrm{GeV} / \mathrm{c}$ to $10 \mathrm{GeV} / \mathrm{c}$ and $C_{4} F_{10}$ gas for medium momenta range up to $70 \mathrm{GeV} / \mathrm{c}$. The $\underline{\mathrm{RICH} 2}$ downstream the magnet has $C F_{4}$ gas radiator and covers high momenta range up to $100 \mathrm{GeV} / \mathrm{c}$. The Čerenkov light is readout by hybrid photon detectors.

The CALO consists of four sub-detectors of interleaved absorber / scintillator design with optical fiber readout. The first two sub-detectors - the scintillator pad detector (SPD) and the preshower detector (PS) - are mono-layers of scintillator tiles, separated by a lead plate of absorber. The SPD is used for early e $/ \gamma$ discrimination, while the PS is important for e $/ \pi$ separation. They are followed by the electromagnetic calorimeter with an energy resolution $\sigma_{E} / E(\%)=9 / \sqrt{E} \oplus 1$ and by the hadron calorimeter, the latter is only used to provide trigger signal. The present accuracy of the calibration is $2 \%$ for the electromagnetic part and $3 \%$ for the hadronic part.

The muon system comprises five tracking stations M1 $\div$ M5. M1 is located in front of the SPD and its goal is to improve the transverse momentum measurements in the trigger system. M $\div$ M5, which are interleaved with iron absorber, reside downstream the CALO. All stations are made of multi-wire proportional chambers except for the inner area of M1 where GEM chambers are used to cope with aging effects due to higher occupancies. The efficiency for $J / \Psi \rightarrow \mu \mu$ decays is found to be $97 \%$ for muons with momentum higher than $4 \mathrm{GeV} / \mathrm{c}$, which is in good agreement with Monte Carlo predictions. For energetic muons ( $>20 \mathrm{GeV} / \mathrm{c}) \mu / \pi$ and $\mu / K$ misidentification rates are below one per cent. 


\subsection{Trigger System}

The LHCb trigger system is organized in two levels. Level-0 or L0 is fully synchronous with accelerator cycle and is hardware-based. It exploits data from the calorimeter, the muon and the pile-up systems to identify events containing high- $p_{T}$ particles, reducing input $40 \mathrm{MHz}$ rate up to $1 \mathrm{MHz}$. The selected events are processed asynchronously through the High Level Trigger (HLT), which software runs on dedicated $\sim 1.5$ thousand CPU farm. The analysis is done on the basis of reconstructed events; the interesting final states are selected using flexible inclusive and exclusive criteria to adapt to changing running conditions and to optimize the physics yield. The output rate of the trigger system is about $3 \mathrm{kHz}$, while overall $\mathrm{L} 0 \times \mathrm{HLT}$ efficiency ranges from $30 \%$ for multibody hadronic channel up to $90 \%$ in case of dimuon selection.

\subsection{LHCb operation in $2010 / 2011$}

During the first half of 2010 the LHCb trigger system ran with loose criteria favorable for the studies of hadronic $B$-decays and for charm physics. From summer on, the selection scheme was re-adjusted back to nominal settings which were optimal for $b$-physics.

So far, LHC has operated at half of the design energy and its filling scheme has had significantly decreased number of bunches. Therefore, in order to collect reasonable statistics, LHCb had to run with increased luminosity $\left(3.5 \times 10^{32} \mathrm{~cm}^{-2} \mathrm{~s}^{-1}\right)$ and increased $\mu(\leq 1.5)$. Even in this challenging environment $\mathrm{LHCb}$ showed robust performance with an average efficiency of $90 \%$ (2011). The overall recorded statistics is $38 \mathrm{pb}^{-1}$ in 2010 and $1.1 \mathrm{fb}^{-1}$ in 2011.

\section{Selected Physics Results}

Certain prerequisite parameters have to be known prior to any further absolute measurements in the charm and the beauty sectors. One of them is the heavy quark-antiquark production crosssections, which were determined at LHCb with early 2010 data. The $b \bar{b}$ cross-section was measured with two methods: (i) using the fraction of $J / \Psi$ from $b$-decays [3] and (ii) on the basis of measured yields of $b$-decays into final states with $D^{0}$ and muon [4]. The cross-section values $\sigma(p p \rightarrow b \bar{b} X)$ extrapolated to the full solid angle are $288 \pm 4 \pm 44 \mu \mathrm{b}$ and $284 \pm 20 \pm 49 \mu \mathrm{b}$ respectively and they are in good agreement with theoretical predictions. The $c \bar{c}$ cross-section was measured from yields of $D^{0}, D^{+}, D^{*+}$ and $D_{s}^{+}$decays and found to be $\sigma(p p \rightarrow c \bar{c} X)=6.10 \pm 0.93 \mathrm{mb}$ which is $\sim 20$ times higher than for $b \bar{b}$ production [5].

Another important parameter is the ratio of strange $B$-meson to light $B$-meson production $f_{s} /\left(f_{u}+f_{d}\right)$ which is important for the determination of any absolute branching ratio of strange $B$-decays. LHCb has measured it using three methods. The first one exploits semileptonic decays of $b$-hadrons, identified by the detection of a muon and a charmed hadron, while the second/third ones employ decay modes $\bar{B}_{s}^{0} \rightarrow D_{s}^{+} \pi^{-}$and $\bar{B}^{0} \rightarrow D^{+} \pi^{-} / \bar{B}^{0} \rightarrow D^{+} K^{-}$. The three results are well compatible with each over and the combined LHCb result is $f_{s} / f_{d}=0.267_{-0.020}^{+0.021}$ assuming the equivalence of $u$ and $d$ quarks. No dependence on $p_{T}$ or rapidity has been observed [6].

\subsection{Searches of new physics via rare $B$-decays}

LHCb uses the approach complementary to the one of ATLAS / CMS experiments and looks for deviations from the SM in known decays due to indirect contributions of new states, mainly via 
loop diagrams. In this way higher energy scales can be accessed as compared with direct searches, which are limited by LHC energy.

Rare decays $B^{0} \rightarrow \mu^{+} \mu^{-}$and $B_{s}^{0} \rightarrow \mu^{+} \mu^{-}$are Flavour Changing Neutral Current processes which can occur through higher order diagrams and therefore they are strongly suppressed in the Standard Model. Its predictions for their branching ratios are precisely calculated:

$$
\mathscr{B}\left(B^{0} \rightarrow \mu^{+} \mu^{-}\right)=(0.1 \pm 0.01) \times 10^{-9}
$$

and

$$
\mathscr{B}\left(B_{s}^{0} \rightarrow \mu^{+} \mu^{-}\right)=(3.2 \pm 0.2) \times 10^{-9},
$$

so any enhancement with respect to these values could come from new physics. The LHCb analysis, performed on the basis of the 2010 dataset and about one third of the 2011 statistics, sets upper limits

$$
\mathscr{B}\left(B_{s}^{0} \rightarrow \mu^{+} \mu^{-}\right)<1.4 \times 10^{-8}
$$

and

$$
\mathscr{B}\left(B^{0} \rightarrow \mu^{+} \mu^{-}\right)<3.2 \times 10^{-9}
$$

at $95 \%$ C.L. [7].

Another promising rare decay is $B^{0} \rightarrow K^{* 0} \mu^{+} \mu^{-}$. The main contribution here comes from electroweak box and penguin diagrams; new states can enter competitive loop diagrams and result in large deviations from SM predictions. LHCb concentrated on three observables potentially sensitive to such effects: (i) the forward-backward asymmetry of the dimuon system $A_{F B}$, (ii) the fraction of longitudinal polarization of the $K^{* 0}$ and (iii) the differential branching fraction as a function of the di-muon invariant mass squared. All these observables were extracted via analysis of angular distributions. So far, LHCb measurements on the basis of one third of the 2011 statistics show good agreement with SM predictions as well as with Babar and Belle results. In particular, the asymmetry value $A_{F B}=-0.10 \pm 0.14 \pm 0.05$ is compatible with zero, which is expected from the SM [8].

The third interesting topic is radiative penguin decays $B^{0} \rightarrow K^{* 0} \gamma$ and $B_{s}^{0} \rightarrow \phi \gamma$, where several observables are supposed to be strongly affected by new physics, e.g. branching ratio, isospin asymmetry and photon polarization. At present, $\mathrm{LHCb}$ has determined the ratio of branching ratios using one third of the 2011 statistics [9]. The resulting value is $\mathscr{B}\left(B^{0} \rightarrow K^{* 0} \gamma\right) / \mathscr{B}\left(B_{s}^{0} \rightarrow \phi \gamma\right)=$ $1.52 \pm 0.14 \pm 0.10 \pm 0.12$, where the last error comes from the precision of $f_{s} / f_{d}$ measurement. The result is within 1.6 standard deviations from the SM prediction. In order to access other observables $1 \div 2 \mathrm{fb}^{-1}$ of statistics is needed.

\subsection{Studies of $C P$-violation in $B^{0}$ - and $B_{s}^{0}$-systems}

LHCb has measured direct $C P$-violation in charmless neutral $B$-decays with $K \pi$ final states. It manifests itself as non-zero value of $C P$-asymmetry defined in terms of decay rates:

$$
A_{C P}\left(B^{0} \rightarrow K \pi\right)=\frac{\Gamma\left(B^{0} \rightarrow K^{+} \pi^{-}\right)-\Gamma\left(\bar{B}^{0} \rightarrow K^{-} \pi^{+}\right)}{\Gamma\left(B^{0} \rightarrow K^{+} \pi^{-}\right)+\Gamma\left(\bar{B}^{0} \rightarrow K^{-} \pi^{+}\right)}=0.088 \pm 0.011 \pm 0.008
$$




$$
A_{C P}\left(B_{s}^{0} \rightarrow K \pi\right)=\frac{\Gamma\left(\bar{B}_{s}^{0} \rightarrow K^{+} \pi^{-}\right)-\Gamma\left(B_{s}^{0} \rightarrow K^{-} \pi^{+}\right)}{\Gamma\left(\bar{B}_{s}^{0} \rightarrow K^{+} \pi^{-}\right)+\Gamma\left(B_{s}^{0} \rightarrow K^{-} \pi^{+}\right)}=0.27 \pm 0.08 \pm 0.02
$$

Both $A_{C P}\left(B^{0} \rightarrow K \pi\right)$ and $A_{C P}\left(B_{s}^{0} \rightarrow K \pi\right)$ are in good agreement with results published by other experiments and represent the most accurate measurements done by a single experiment [10].

One of the most important topics of the LHCb physics program is the indirect time dependent $C P$-violation in $B_{s}^{0}$-system, when decay to the same final state can occur either directly or via intermediate $B_{s}^{0}-\bar{B}_{s}^{0}$ transition due to the mixing. It is characterized by $C P$-violating phase $\phi_{s}$, where new physics can provide an additional contribution. The golden channel for such studies is recognized to be $B_{s}^{0} \rightarrow J / \Psi \phi$ and its $\phi_{s}$-phase is precisely calculated in the SM: $\phi_{s}=-0.036 \pm 0.002 \mathrm{rad}$. This is a vector-vector final state and therefore $\mathrm{P}$ - and $\mathrm{S}$-waves contributions have to be disentangled which implies additional angular analysis. For that reason the set of fit parameters includes three complex angular amplitudes at $t=0$ to describe P-wave $\left(A_{0}, A_{\|}, A_{\perp}\right)$ and one complex angular amplitude at $t=0$ for $\mathrm{S}$-wave $\left(A_{s}\right)$. The remaining parameters are the phase $\phi_{s}$ itself, the average $B_{s}^{0}$-decay width $\Gamma_{s}$ and the decay width difference between $B_{s}^{0}$ mass eigenstates $\Delta \Gamma_{s}$. The oscillation frequency $\Delta m_{s}$ was fixed to the value $17.63 \pm 0.11 \pm 0.02 \mathrm{ps}^{-1}$ previously measured by LHCb [11].

The decay rates are invariant under the following simultaneous transformation:

$$
\left(\phi_{s}, \Delta \Gamma_{s}, \delta_{\|}-\delta_{0}, \delta_{\perp}-\delta_{0}, \delta_{s}-\delta_{0}\right) \leftrightarrow\left(\pi-\phi_{s},-\Delta \Gamma_{s}, \delta_{0}-\delta_{\|}, \pi+\delta_{0}-\delta_{\perp}, \delta_{0}-\delta_{s}\right),
$$

where $\delta$ are phases of angular amplitudes $A$, and therefore $\phi_{s}$ value is two-fold ambiguous. This uncertainty can be resolved via the measurement of the phase of the S-wave contribution as a function of $K K$ invariant mass from $\phi$ decay [12], but the work is still in progress.

Another decay mode, beneficial to $\phi_{s}$ determination, is $B_{s}^{0} \rightarrow J / \Psi f_{0}(980)$. LHCb was the first experiment to observe it experimentally in 2011 [13]. Unlike the golden channel, the final state here is $C P$-odd eigenstate and only $\mathrm{S}$-wave contributes, which allows to lessen the number of parameters for the fit. However, it also means that $\Gamma_{s}$ and $\Delta \Gamma_{s}$ can not be determined simultaneously, therefore at least one of them had to be fixed to the value measured via the golden channel.

The outcome of $B_{s}^{0} \rightarrow J / \Psi \phi$ channel studies is reported in [2], while analysis of $\bar{B}_{s}^{0} \rightarrow J / \Psi f_{0}$ is published in [14]. Combined results for $\phi_{s}$ are discussed in [15]. The values of $\phi_{s}$ obtained by LHCb using various methods are well consistent; the combined result is $\phi_{s}=-0.03 \pm 0.16 \pm 0.07 \mathrm{rad}$. Besides, LHCb provided the world most precise measurement of $\Gamma_{s}=0.656 \pm 0.009 \pm 0.008 \mathrm{ps}^{-1}$ and the first evidence of non-zero $\Delta \Gamma_{s}=0.123 \pm 0.029 \pm 0.011 \mathrm{ps}^{-1}$ [2].

\subsection{Studies of $C P$-violation in the charm sector}

One of the most interesting $\mathrm{LHCb}$ results in the charm sector comes from the measurements of the time-integrated $C P$-asymmetries $A_{C P}$ of $D^{0} \rightarrow h^{+} h^{-}$decay rates. $A_{C P}$ is made up of the direct component $a_{C P}^{d i r}$ related to the $C P$-violation in the decay amplitudes and of the indirect component $a_{C P}^{i n d i r}$ associated with $C P$-violation in the mixing as well as with the interference between decay and mixing diagrams. According to the SM, the main contribution comes from $a_{C P}^{d i r}$, which could be on the level of $O\left(10^{-3}\right)$ or less depending on the final state, but physics beyond the SM could contribute to both direct and indirect parts. 
Using about $0.62 \mathrm{fb}^{-1}$ of the 2011 statistics, $\mathrm{LHCb}$ performed the measurement of the difference $\Delta A_{C P}$ between integrated $C P$-asymmetries of $D^{0} \rightarrow K^{+} K^{-}$and $D^{0} \rightarrow \pi^{+} \pi^{-}$decays with $D^{0}$ reconstructed as a part of $D^{*+} \rightarrow D^{0} \pi^{+}$chain in order to tag its initial flavour state. In this difference, all instrumental asymmetries cancel and the experimental $\Delta A_{C P}$ is equal to the difference of intrinsic physics $C P$-asymmetries. The obtained value $\Delta A_{C P}(\%)=-0.82 \pm 0.21 \pm 0.11$ differs from the hypothesis of $C P$ conservation by 3.5 standard deviations [16] and this is the first evidence of $C P$-violation in the charm sector.

The intrinsic $C P$-asymmetry can be approximated as $A_{C P} \approx a_{C P}^{d i r}+a_{C P}^{i n d i r} \times \tau / t$, where $t$ is true $D^{0}$ lifetime and $\tau$ is the average proper time in the sample used. For LHCb $\left(\tau_{K K}-\tau_{\pi \pi}\right) / t$ was measured to be $(9.83 \pm 0.22 \pm 0.19) \%[16]$ and therefore the contribution of the indirect component mostly cancels.

\section{4 $\mathrm{W}$ and $\mathrm{Z}$ bosons production}

The theoretical predictions for $\mathrm{W} / \mathrm{Z}$ production cross-sections have up to $10 \%$ uncertainty, coming mostly from parton distribution functions (PDFs). LHCb measurements can provide an important input to constrain PDFs, especially in the region of rapidities $\eta>2.5$ which is not covered by ATLAS and CMS. LHCb has studied $Z \rightarrow \mu \mu$ and $W \rightarrow \mu v$ decay modes with the full 2010 statistics [17] and $Z \rightarrow \tau \tau$ channel using the full 2010 dataset and about a quarter of the 2011 statistics [18]. The $\mathrm{W} / \mathrm{Z}$ production cross-sections, their ratios and $\mathrm{W}$ charge asymmetry have been obtained; all of them are found to be consistent with NNLO predictions. The measured ratio $\sigma(Z \rightarrow \tau \tau) / \sigma(Z \rightarrow \mu \mu)=1.09 \pm 0.17$ is consistent with lepton universality.

\section{Conclusions}

The data taking period of 2010/2011 has been extremely fruitful for the LHCb experiment which demonstrated very good detector and trigger performances. Using only a fraction of the full statistics, LHCb has obtained results already competitive with Tevatron and B-factories and has performed several of the most precise measurements in the world. So far, no deviations from the SM have been observed. Once the full statistics processed, it will be possible to improve the accuracy of current measurements and to access to other interesting processes and observables.

\section{References}

[1] LHCb Collab., The LHCb Detector at the LHC, JINST 3,S08005 (2008).

[2] LHCb Collab., Tagged time-dependent angular analysis of $B_{s}^{0} \rightarrow J / \Psi \phi$ decays with $337 \mathrm{pb}^{-1}$ at LHCb, LHCb-CONF-2011-049, CERN, Geneva, 2011.

[3] LHCb Collab., Measurement of $J / \Psi$ production in pp collisions at $\sqrt{s}=7 \mathrm{TeV}$, Eur. Phys. J. C 71, 1645 (2011).

[4] LHCb Collab., Measurement of $\sigma(p p \rightarrow b \bar{b} X)$ at $\sqrt{s}=7 \mathrm{TeV}$ in the forward region, Phys. Lett. B 694, 209 (2010).

[5] LHCb Collab., Prompt charm production in pp collisions at $\sqrt{s}=7$ TeV, LHCb-CONF-2010-013, CERN, Geneva, 2010. 
[6] LHCb Collab., Measurement of b hadron production fractions in $7 \mathrm{TeV}$ pp collisions, arXiv:1111.2357 [hep-ex].

[7] LHCb Collab., Search for rare decays $B_{s}^{0} \rightarrow \mu^{+} \mu^{-}$and $B^{0} \rightarrow \mu^{+} \mu^{-}$, arXiv:1112.1600 [hep-ex].

[8] LHCb Collab., Angular analysis of $B^{0} \rightarrow K^{* 0} \mu^{+} \mu^{-}$, LHCb-CONF-2011-038, CERN, Geneva, 2011.

[9] LHCb Collab., Measurement of the ratio of branching fractions $\mathscr{B}\left(B^{0} \rightarrow K^{* 0} \gamma\right) / \mathscr{B}\left(B_{s}^{0} \rightarrow \phi \gamma\right)$ with the LHCb experiment at $\sqrt{s}=7 \mathrm{TeV}$, LHCb-CONF-2011-055, CERN, Geneva, 2011.

[10] LHCb Collab., Charmless charged two-body B-decays at LHCb with 2011 data, LHCb-CONF-2011-042, CERN, Geneva, 2011.

[11] LHCb Collab., Measurement of the $B_{s}^{0}-\bar{B}_{s}^{0}$ oscillation frequency $\Delta m_{s}$ in $B_{s}^{0} \rightarrow D_{s}^{-}(3) \pi$ decays, arXiv:1112.4311 [hep-ex].

[12] Y. Xie et al., JHEP 0909 354, 074 (2009).

[13] LHCb Collab., First observation of $B_{s} \rightarrow J / \Psi f_{0}(980)$ decays, Phys. Lett. B 698, 115 (2011).

[14] LHCb Collab., Measurement of the $C P$-violating phase $\phi_{s}$ in $\bar{B}_{s}^{0} \rightarrow J / \Psi f_{0}(980)$, Phys. Lett. B 707, 497 (2012).

[15] LHCb Collab., Combination of $\phi_{s}$ measurements from $B_{s}^{0} \rightarrow J / \Psi \phi$ and $B_{s}^{0} \rightarrow J / \Psi f_{0}(980)$, LHCb-CONF-2011-056, CERN, Geneva, 2011.

[16] LHCb Collab., Evidence for CP-violation in time-integrated $D^{0} \rightarrow h^{-} h^{+}$decay rates, arXiv:1112.0938 [hep-ex].

[17] LHCb Collab., Updated measurements of $W$ and $Z$ production at $\sqrt{s}=7 \mathrm{TeV}$ with the LHCb experiment, LHCb-CONF-2011-039, CERN, Geneva, 2011.

[18] LHCb Collab., Z cross-section measurement at $\sqrt{s}=7$ TeV using the channel $Z \rightarrow \tau \tau$, LHCb-CONF-2011-041. 